\title{
Preoperative $B R A F$ inhibition in patients with irresectable locally advanced stage III melanoma
}

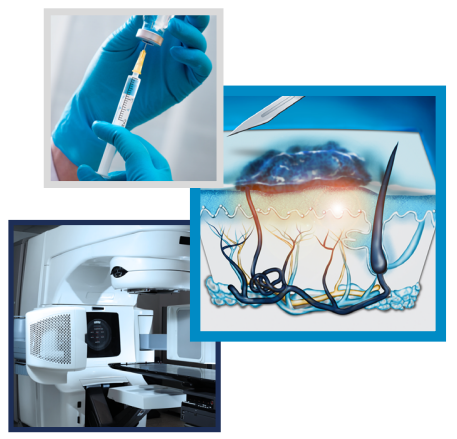

\author{
Marloes Faut ${ }^{1}$, Mathilde Jalving ${ }^{2}$, Gilles F Diercks ${ }^{3}$, Geke A Hospers², Barbara L van \\ Leeuwen ${ }^{1}$ \& Lukas B Been*,1 \\ ${ }^{1}$ Department of Surgical Oncology, Department of Surgery, University of Groningen, University Medical Center Groningen, \\ Hanzeplein 1, P.O. Box 30.001, 9700RB Groningen, The Netherlands \\ ${ }^{2}$ Department of Medical Oncology, University of Groningen, University Medical Center Groningen, Hanzeplein 1, P.O. Box 30.001, \\ 9700RB Groningen, The Netherlands \\ ${ }^{3}$ Department of Pathology, University of Groningen, University Medical Center Groningen, Hanzeplein 1, P.O. Box 30.001, 9700RB \\ Groningen, The Netherlands \\ *Author for correspondence: Tel.: +315 0361 2317; Fax: +315 0361 1745; I.b.been@umcg.nl
}

\section{Practice points}

- Clinically evident resectable stage III melanoma is preferably treated by a surgical resection as neoadjuvant treatment is not an established treatment modality (yet) for stage III melanoma. After surgical resection, adjuvant therapy can be considered for these patients.

- When confronted with advanced stage III melanoma, full body imaging with fluorodeoxyglucose positron emission tomography-computed tomography or CT-scan including an MRI of the brain should be performed, to determine the extent of metastases and to exclude stage IV.

- Inoperable melanoma is generally treated as stage IV melanoma and can be treated with targeted therapy and immunotherapy. Neoadjuvant treatment with $B R A F$ inhibitors can facilitate a surgical resection. However, it is only available in the context of clinical trials.

- The duration of neoadjuvant treatment with BRAF inhibitors remains to be determined based on the results of neoadjuvant trials. Surgical resection needs to be planned at the time of maximal response to neoadjuvant treatment.

- With the availability of systemic treatment options, previously inoperable patients can be treated with neoadjuvant $B R A F$ inhibitors, a surgical resection and adjuvant immunotherapy. With the ongoing advancements made in systemic therapy for metastasized melanoma, it is likely that these treatment options continue to improve.

- It is to be determined by clinical trials if neoadjuvant treatment followed by a surgical resection for advanced stage III melanoma improves survival for these patients.

Aim: Neoadjuvant treatment of locally advanced disease with $B R A F$ inhibitors is expected to increase the likelihood of a R0 resection. We present six patients with stage III unresectable melanoma, neoadjuvantly treated with $B R A F$ inhibitors. Methods: Patients with unresectable, BRAF-mutated, stage III melanoma, were treated with BRAF inhibitors between 2012 and 2015. Unresectability was determined based on clinical and/or radiological findings. At maximal response, resection was performed. The specimen was reviewed to determine the degree of response. Results: In five of six patients a radical resection was achieved. Postoperative complications were unremarkable. In five of six resected specimens, vital tumor tissue was found. Conclusion: Neoadjuvant BRAF inhibitor treatment of locally advanced melanoma is feasible and has the potential to facilitate an $\mathrm{RO}$ resection.

First draft submitted: 17 January 2018; Accepted for publication: 19 April 2018; Published online: 16 May 2018

Keywords: BRAF inhibitor • melanoma • neoadjuvant • surgical resection • unresectable

For stage III melanoma patients, 5-year overall survival is associated with tumor burden and ranges between 30 and $80 \%$ [1]. It is well established that a radical resection of stage III melanoma is prognostically favorable compared with an R1 resection. In some cases, an R0 resection is not possible due to tumor size and/or adherent vital structures

Future : Medicine 
such as neurovascular bundles impeding radical surgical treatment. In cases where stage III melanoma is deemed unresectable, patients are historically treated in a similar fashion to stage IV patients. Since the introduction of targeted therapy and immune checkpoint inhibitors, the prognoses for patients with unresectable stage III and IV melanoma have improved [2-6]. Approximately 50\% of cutaneous melanomas harbor a $B R A F$ mutation [7]. These patients can be treated with a $B R A F$ inhibitor, alone or in combination with an $M E K$ inhibitor. This results in exceptionally fast and extensive responses in approximately $50 \%$ of patients, within 6 weeks $[3,8-9]$. Median response duration for vemurafenib is 6.7 months, and 5.1 months for patients receiving dabrafenib $[8,9]$. The addition of an $M E K$ inhibitor prolongs progression-free survival to a median of 9.3 months [4]. Using BRAF inhibitors as an induction treatment to reduce tumor size in unresectable stage III melanoma, paving the way for a radical surgical resection, is a logical next step. We present data on six unresectable stage III melanoma patients treated with $B R A F$ inhibition neoadjuvantly in order to facilitate a surgical resection, at our center. To determine the response to $B R A F$ inhibitor treatment, a grading system was created. The aim of this study was to describe the feasibility and pitfalls of this treatment approach.

\section{Materials \& methods \\ Study population}

The population consisted of patients with locally advanced stage III melanoma that was deemed either unresectable due to encasement of adherent structures such as arteries, veins or nerves, or due to the mutilating nature of a resection. This study was conducted at the University Medical Center Groningen. This is a university hospital and tertiary referral center in the northern part of the Netherlands with a catchment area of 1.5 million inhabitants. Patients were included between 2012 and 2015. All patients tested positive for a therapy responsive BRAF mutation and had no history of prior $B R A F$ inhibitor treatment. Locally advanced stage III melanoma was deemed unresectable based on clinical and/or radiological evaluation and after discussion during a multidisciplinary tumor board meeting. This multidisciplinary panel consisted of at least one surgical oncologist, radiologist (or nuclear medicine physician), medical oncologist, radiotherapist, dermatologist, pathologist and a neurologist. In all patients a fluorine18 fluorodeoxyglucose positron emission tomography combined with a diagnostic contrast-enhanced CT scan of thorax and abdomen was performed, to exclude stage IV melanoma prior to start BRAF inhibitor treatment.

\section{Study design}

After medical evaluation and informed consent to the treatment plan, $B R A F$ inhibitor treatment was commenced. Patients were treated with $B R A F$ inhibition and, based on availability, combined with $M E K$ inhibition. Monotherapy was the only available therapy as standard of care in the Netherlands before 2015. From mid-2015 onwards, combined dabrafenib and trametinib were available as standard of care.

Physical examination was performed at every outpatient clinic visit (every 2-4 weeks). Response evaluation by imaging was usually performed after 2 months of $B R A F$ inhibitor treatment. This interval was prolonged if it was clinically evident that surgical resection could not be performed at that time and $B R A F$ inhibitor treatment was tolerated well. Patients were treated until maximal response to $B R A F$ inhibitor treatment. Maximal response was reached if there was no longer evidence of diminishing tumor size either by clinical or radiological examination. Resection was planned within 6 weeks of maximum response. Postoperative morbidity and mortality were assessed during a 30-day follow-up period. R0 resection was defined as a complete resection with tumor-free resection margins. After the surgical resection, follow-up was conducted by the surgical oncologist every 3 months by physical examination, serum LDH and S-100B levels and imaging when indicated.

\section{Outcomes}

Data were collected concerning patient characteristics, treatment regimen and treatment duration. Toxicity of neoadjuvant treatment was assessed at every outpatient clinic visit (every 2-4 weeks) and was retrospectively graded according to the Common Terminology Criteria for Adverse Events (CTCAE) version 4.0 by evaluation of the electronic health records [10]. Histological sampling to determine BRAF mutation status was either performed on the primary tumor or a metastasis. After histological sampling, DNA extraction was performed using Cobas extraction-kit, Roche ${ }^{\odot}$. BRAF mutation analysis prior to September 2014 was performed using HRM screening and confirmation with Sanger sequence analysis. After September 2014, multiplex PCR and PGM/Ion-Torrent sequence analysis containing the following genes: ALK, BRAF, EGFR, ERBB2, GNA11, GNAQ, KIT, KRAS, NRAS, PDGFRA and PIK3CA was performed. Pathology specimens were reviewed by a melanoma pathologist, in particular 
Table 1. Response grading system to BRAF inhibitor treatment.

\section{Response}

No response

Partial response

Complete response

Mixed response

\section{Explanation}

No reduction of vital tumor cells in the resected specimen

Melanophages with fibrosis and/or necrosis with remaining vital tumor cells in the resected specimen

No vital tumor cells identifiable. Only melanophages with fibrosis and/or necrosis in the resected specimen

A combination of no response and/or partial response and/or complete response in the resected specimen

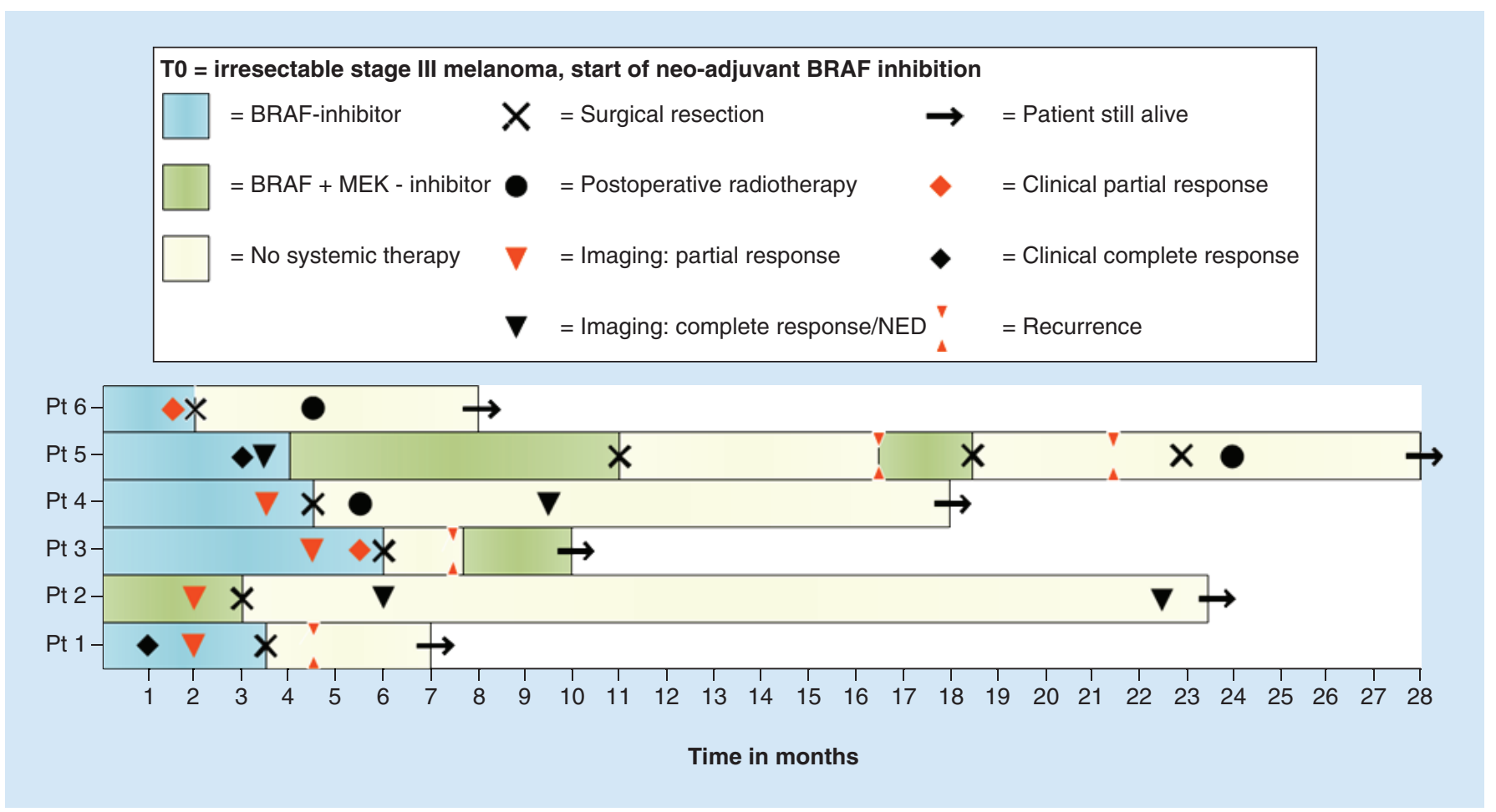

Figure 1. Swimmers plot of all patients.

with respect to the estimated percentage of fibrosis with melanophages, necrosis and the percentage of vital tumor tissue in the specimen. A grading system for response to $B R A F$ inhibitor treatment was created based on the percentage of vital tumor tissue, fibrosis, melanophages and/or necrosis in the resected specimen (Table 1).

\section{Statistical analysis}

Descriptive statistics were performed using IBM SPSS statistics, version 22.

\section{Results}

\section{Patient \& tumor characteristics}

Six patients were treated neoadjuvantly with $B R A F$ inhibitors between January 2012 and December 2015. One patient presented with unresectable melanoma at the time of the primary diagnosis. The other five patients presented with unresectable local disease after treatment of the primary melanoma, with a median interval of 60 months (range 2-100, Figure 1). The patient and tumor characteristics and treatment regimen are shown in Table 2.

\section{$B R A F$ inhibitor therapy}

Patients were treated with $B R A F$ inhibitors during a median of 3.8 (range 2-11) months (Table 3). Five of six patients experienced toxicity of $B R A F$ inhibitor treatment, mainly grade 1 palmar-plantar erythrodysesthesia 


\begin{tabular}{|c|c|c|c|c|c|c|c|c|}
\hline Patient & $\mathrm{Age}^{\dagger}$ & Gender & Site primary & Stage primary & Time $^{\ddagger}$ & Metastatic site & $\begin{array}{l}\text { Reason } \\
\text { irresectable }\end{array}$ & Therapy of choice \\
\hline 1 & 37 & Female & $\begin{array}{l}\text { Lower extremity } \\
\text { (left) }\end{array}$ & pT1BN1a & 63 & $\begin{array}{l}\text { Inguinal and } \\
\text { iliac lymph } \\
\text { nodes }\end{array}$ & $\begin{array}{l}\text { Encasement of } \\
\text { adherent } \\
\text { structures }\end{array}$ & $\begin{array}{l}\text { Dabrafenib } \\
150 \mathrm{mg} \text { twice daily }\end{array}$ \\
\hline 2 & 36 & Female & $\begin{array}{l}\text { Lower extremity } \\
\text { (right) }\end{array}$ & pT3bN1a & 60 & $\begin{array}{l}\text { Iliac and } \\
\text { para-aortal } \\
\text { lymph nodes }\end{array}$ & $\begin{array}{l}\text { Encasement of } \\
\text { adherent } \\
\text { structures }\end{array}$ & $\begin{array}{l}\text { Dabrafenib } \\
150 \text { mg twice } \\
\text { daily + trametinib } \\
2 \text { mg once daily }\end{array}$ \\
\hline 3 & 66 & Female & $\begin{array}{l}\text { Lower extremity } \\
\text { (right) }\end{array}$ & pT4bN2b & 2 & $\begin{array}{l}\text { Right gluteal } \\
\text { region and } \\
\text { ilioinguinal } \\
\text { nodal disease }\end{array}$ & $\begin{array}{l}\text { Encasement of } \\
\text { adherent } \\
\text { structures and } \\
\text { due to } \\
\text { mutilating } \\
\text { nature of } \\
\text { resection }\end{array}$ & $\begin{array}{l}\text { Dabrafenib } \\
150 \text { mg twice daily }\end{array}$ \\
\hline 6 & 49 & Male & $\begin{array}{l}\text { Head \& neck } \\
\text { (right cheek) }\end{array}$ & pT3aN1a & 6 & $\begin{array}{l}\text { Locoregional } \\
\text { and regional } \\
\text { nodal disease }\end{array}$ & $\begin{array}{l}\text { Mutilating } \\
\text { nature of } \\
\text { resection }\end{array}$ & $\begin{array}{l}\text { Dabrafenib } \\
150 \mathrm{mg} \text { twice daily }\end{array}$ \\
\hline
\end{tabular}

${ }^{\dagger}$ Age is defined as age at presentation with irresectable melanoma.

$¥$ Time is defined as time in months between treatment of primary tumor and detection of locally advanced melanoma.

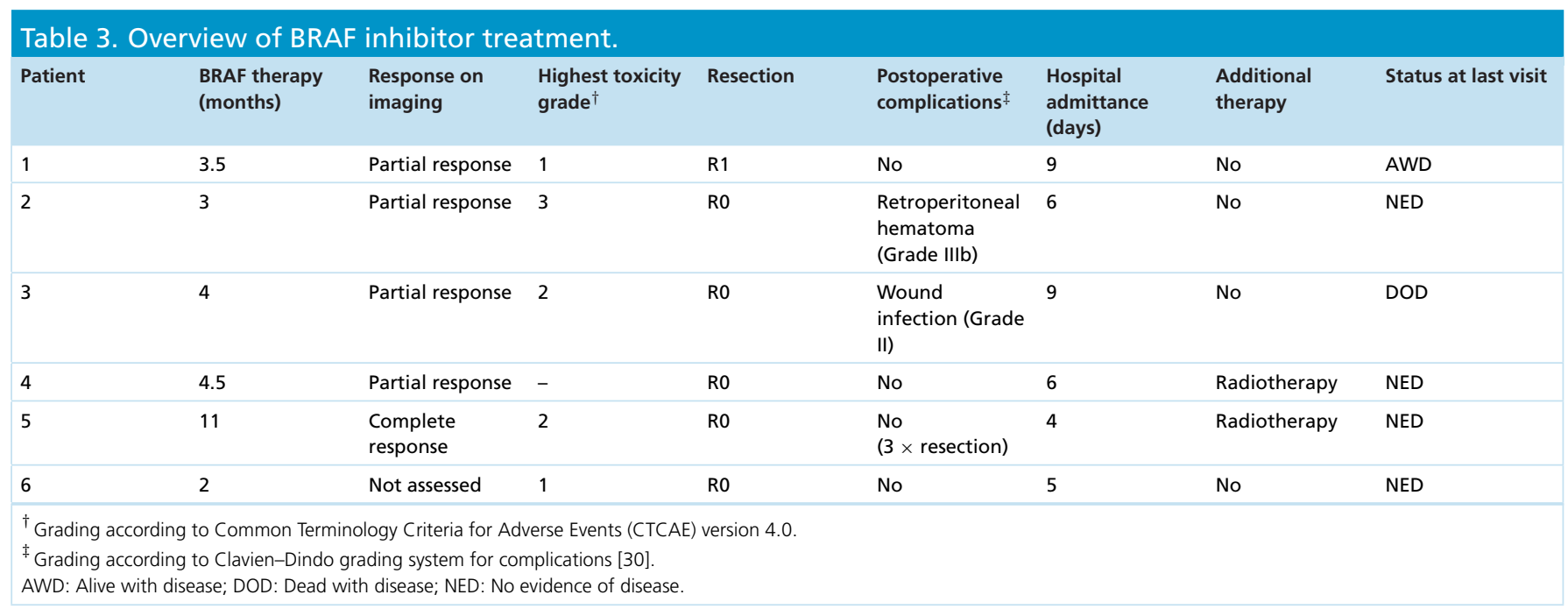

syndrome, headache and grade 2 alopecia. Patient two suffered from grade III headache, for which she was admitted to the hospital. All patients recovered completely after treatment discontinuation.

\section{Surgical resection}

Surgical resection was performed lege artis. Fibrosis of tumor tissue was frequently seen. This added technical difficulty to the procedure. However, this did not lead to surgical complications intraoperatively. An R0 resection was achieved in five patients. Median postoperative hospital stay was 6 days (range 5-9). One patient was readmitted 15 days after discharge due to a retroperitoneal hematoma presenting with fever, abdominal pain, leukocytosis and hydronephrosis. The hematoma was caused by postoperative bleeding and was resolved by re-exploration; the 


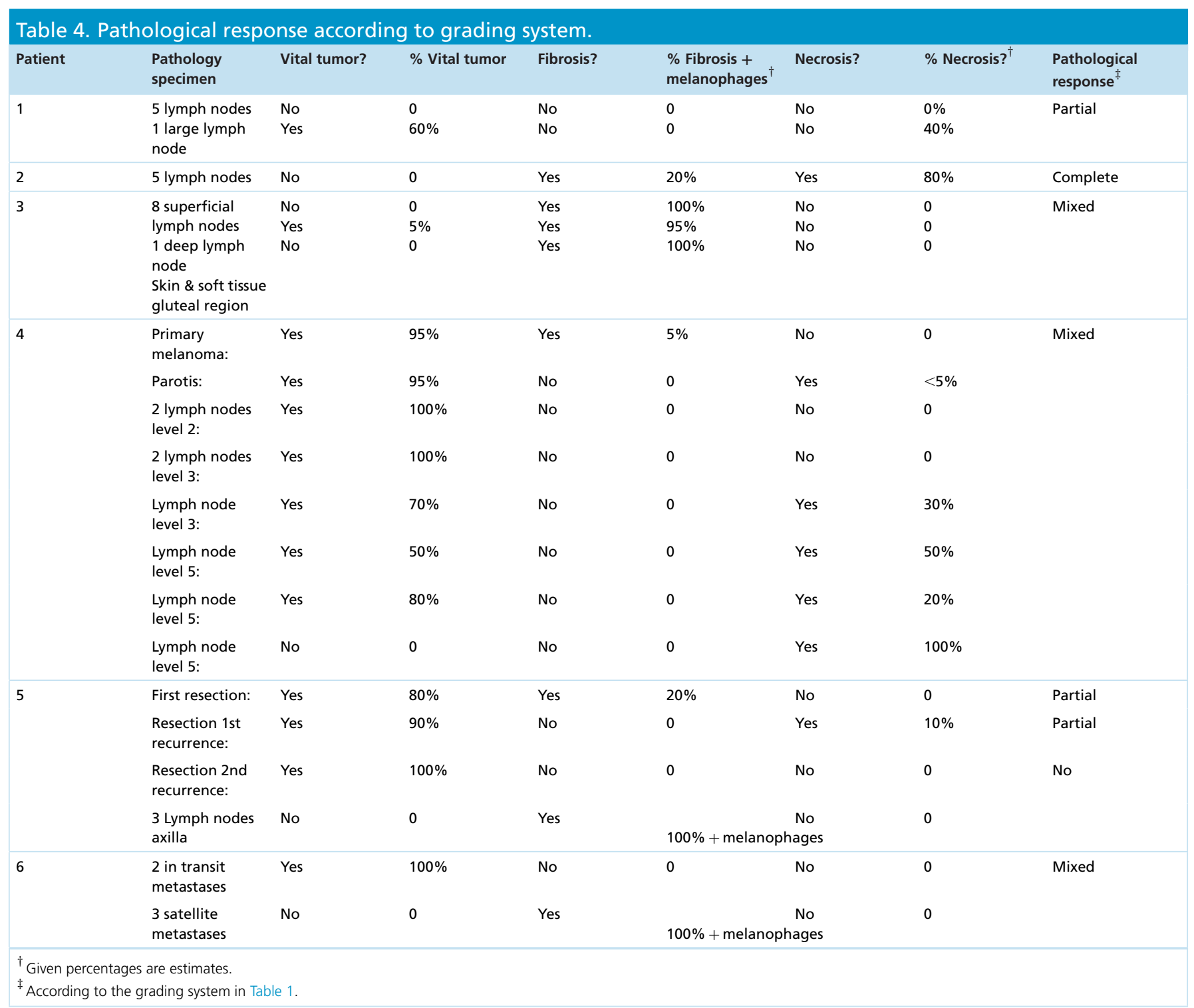

patient recovered fully. Another patient was readmitted 5 days after discharge with a wound infection. This resolved after intravenous administration of antibiotics and negative wound pressure therapy during 6 weeks. The 30-day postoperative period was uncomplicated in the remaining four patients.

\section{Pathological evaluation}

In one patient a complete pathological response was found, the five other resected specimens contained vital tumor tissue (Table 4). The degree of response to $B R A F$ inhibitor treatment varied throughout the different resected specimens within the patients.

\section{Follow-up}

Three patients had a recurrence. In patient one, on imaging, response to $B R A F$ inhibitor treatment was partial. There was diminution of tumor size in some lymph nodes. One week prior to resection $B R A F$ inhibitor treatment was ceased and patient experienced complaints similar to the period prior to $B R A F$ inhibitor treatment (abdominal pain), as well as a rise in S-100B levels, suggestive for a rapid progression. Perioperatively, the iliac lymph nodes encased the artery and vein. A safe procedure was not possible without dissecting tumor tissue. Consequently the tumor was perforated and the resection was irradical. One month after the R1 resection, a fluorine-18 fluorodeoxyglucose positron emission tomography-scan was performed to exclude potential stage IV disease before 


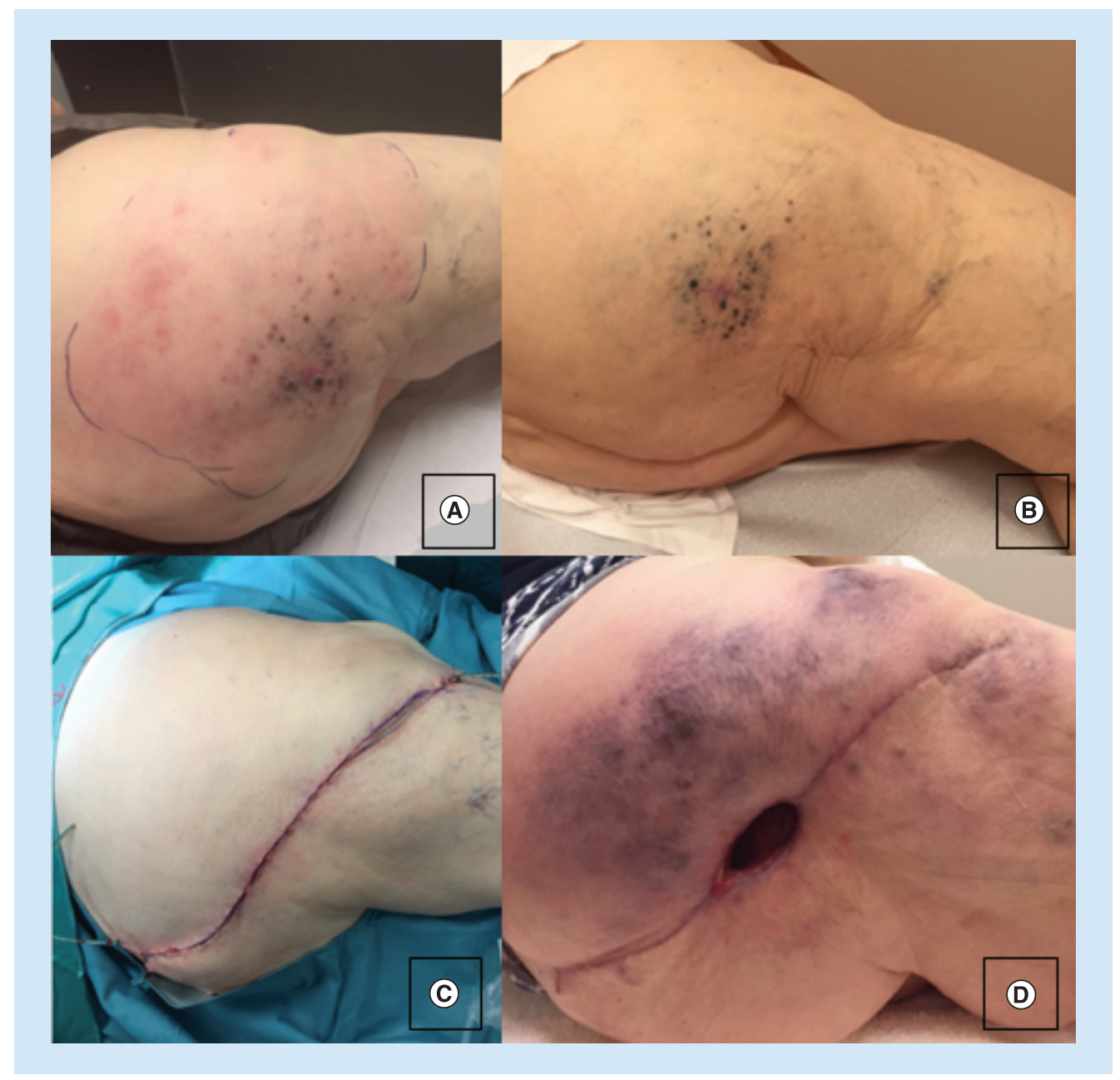

Figure 2. Unresectable satellite metastases and lymphangitis on the right gluteal region. (A) Before treatment; (B) during treatment; (C) after surgical resection; (D) one month after surgical resection.

commencing adjuvant radiation therapy to the groin. A solitary pulmonary metastasis was identified for which surgical resection performed. Adjuvant radiation therapy was no longer indicated. Patient three suffered from a clinically evident local recurrence 1.5 months after R0 resection, with pigmented lymphangitis and satellitosis at the location where previous metastases had disappeared during $B R A F$ inhibitor treatment. Due to the extent of the recurrence and the short disease-free interval, the local recurrence was deemed unresectable. BRAF and $M E K$ inhibition was commenced, but the patient died due to metastatic disease (Figure 2). Patient five had a local recurrence after 5.5 months, this was located submentally, where the previous lymph node metastasis had disappeared during $B R A F$ inhibitor treatment, and a second recurrence five months later. Both recurrences were treated by surgical resection. Due to the multiple resections of submental skin and concurrent reduction of available resection possibilities in case of subsequent recurrence, the last resection was followed by adjuvant radiation. At the time of writing with a median follow-up of 14 months, five patients are alive, and four patients have no evidence of disease. Patients are still in follow-up in the University Medical Center Groningen.

\section{Discussion}

This study shows that preoperative $B R A F$ inhibitor treatment of unresectable stage III melanoma is feasible. Toxicity was minimal and there were few postoperative complications attributable to the neoadjuvant treatment.

In other tumor types, neoadjuvant chemotherapy either as mono treatment or in combination with radiation is an established treatment option, and has proven to be valuable in achieving R0 resections and local control after surgical treatment $[11,12]$. The desire for improved disease-free and overall survivals in melanoma patients 
has led to investigation of neoadjuvant interferon and bevacizumab in patients with high-risk primarily resectable lymph node metastases. Clinical response was seen in approximately $50 \%$ of patients [13,14]. Neither significant improvement of disease-free survival nor overall survival was demonstrated in these studies. None of these studies have focused on unresectable stage III melanoma. Case reports describing successful induction of tumor response with $B R A F$ inhibitors followed by a successful surgical resection in unresectable stage III melanoma are scarce [15-18]. One previously published retrospective patient series describes 15 patients with locoregionally advanced, $B R A F-$ mutated, stage III melanoma. These patients were treated with $B R A F$ inhibitors and six patients had a radical resection of residual disease. None of these patients were treated intentionally in a neoadjuvant fashion. Pathologic responses seen in the resected specimens were comparable to those in our series. The objective response rate was, however, lower, with only six out of 15 patients receiving surgical resection following $B R A F$ inhibitor treatment [19].

Side effects due to $B R A F$ inhibitor treatment in our series were comparable to results described in the literature $[3,9]$.

In this series $B R A F$ inhibitor treatment led to fibrosis of tumor tissue and added a challenge to the surgical procedure itself; however, it did not lead to intraoperative complications in our series. This is compatible with previous reports which do not describe increased postoperative complications after $B R A F$ inhibitor treatment [19,20].

The use of $B R A F$ inhibitors as a single therapy (or combined with an $M E K$ inhibitor) in stage IV and unresectable stage III melanoma is standard of care. When melanomas harbor a therapy responsive $B R A F$ mutation, treatment with $B R A F$ inhibitors leads to objective rapid and impressive responses in $53 \%$ of patients treated with vemurafenib and $50 \%$ of patients treated with dabrafenib. In a small subset of patients $(\sim 20 \%)$ durable responses of $>2$ years on the $B R A F$ and $M E K$ inhibitors combination have been described [21]. The possibility of long-term survival on $B R A F$ inhibitor treatment complicates decisions on timing of surgical procedures after neoadjuvant $B R A F$ inhibitor treatment. This is illustrated by patients three and five who were treated for $>2$ months before surgical resection of locally advanced stage III melanoma was planned (Figure 1). A risk of this long-term $B R A F$ inhibitor treatment, is the possibility of disease progression and the concurrent loss of a surgical window during BRAF inhibitor treatment. This may be preventable by frequent response evaluation. Due to the fast responses seen with $B R A F$ inhibitor treatment, a surgical resection can be planned after only weeks of response to $B R A F$ inhibitor treatment, therefore the treatment period can be relatively short. The risk of disease progression during the first 6 weeks of treatment is approximately three percent [22-24]. Adequate timing of the surgical procedure is of great importance, this remains a challenging and multidisciplinary decision. The differences in treatment duration in this patient series underline this challenge.

Pathological responses to $B R A F$ inhibitor treatment varied throughout all the resected specimens within the patients in this study. Therefore, existing grading systems for neoadjuvant chemotherapy for instance as in breast carcinomas were not applicable, as responses to chemotherapy in breast carcinomas are more similar throughout the resected specimens within the patients [25]. In future neoadjuvant trials, grading systems should describe the percentage of vital tumor tissue throughout the resected specimen. Mixed responses were frequently seen in this study and should be described in future trials.

In this series three out of six patients had a recurrence of which two were local recurrences. Adjuvant radiation therapy decreases the risk of local recurrences after lymph node dissection compared with observation in high-risk stage III melanoma patients (21 vs 36\% relapse) and can also be considered in this patient group [26]. In the future, neoadjuvant treatment followed by resection of advanced stage III melanoma could potentially be followed by adjuvant immunotherapy. The use of adjuvant ipilimumab improves 3-year recurrence-free survival in complete resected stage III melanoma patients compared with adjuvant placebo (46.5 vs $34.8 \%)$ [27,28].

There are several studies for neoadjuvant treatment of resectable stage III melanoma ongoing at this moment (ClinicalTrials.gov identifiers: NCT01972347, NCT02036086, NCT02858921, NCT02303951, Trialregister.nl identifier: NTR4654). These prospective studies will give more insight into response rates and probability of achieving R0 resections. In current and future clinical trials, a definite neoadjuvant treatment period is needed and should be defined, to help determine reproducibility and clinical applicability of data, as well as longer follow-up in larger populations to be able to truly assess long-term clinical benefit.

\section{Conclusion}

This experience with neoadjuvant $B R A F$ inhibitor treatment shows that this treatment is feasible in unresectable stage III melanoma patients. It can lead to resectable stage III melanoma and facilitate an R0 resection in previously unresectable patients. 


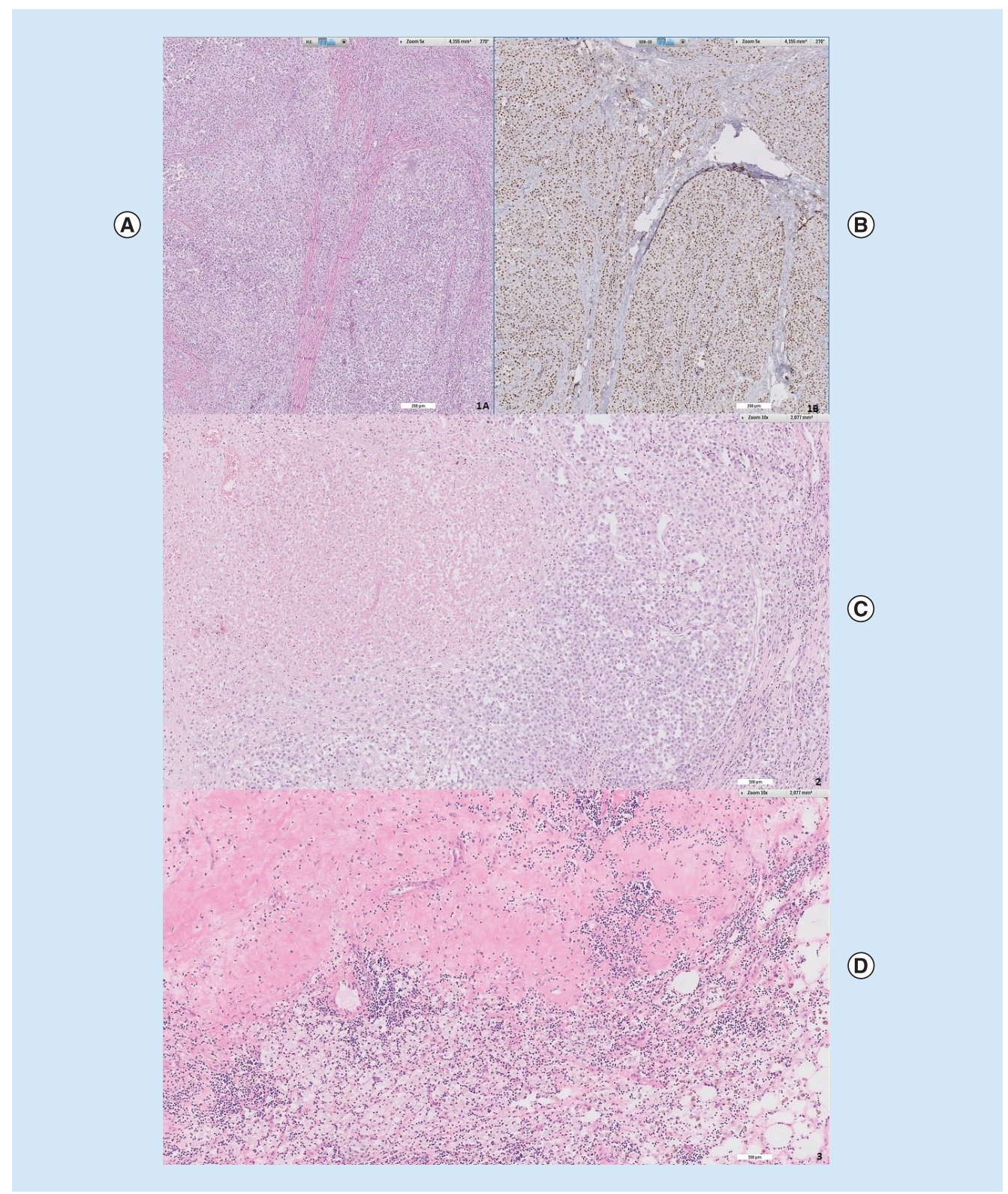

Figure 3. Microscopic images of melanoma treated with neo-adjuvant BRAF-inhibition. (A) No response to BRAF inhibitor treatment; (B) no response to BRAF inhibitor treatment, SOX10 stain; (C) partial response to BRAF inhibitor treatment; (D) complete response to BRAF inhibitor treatment.

\section{Future perspective}

Future research should be aimed at determining which patients benefit from neoadjuvant and adjuvant treatment, in order to be able to determine in which patients treatment benefits outweigh treatment morbidity. In the Netherlands, adjuvant treatment with immunotherapy is only available in clinical trials and not as standard of care. In future trials adjuvant therapy should be strongly considered.

Financial \& competing interests disclosure

GAP Hospers is in the advisory board of Roche and Novartis (payment to the institution). The authors have no other relevant affiliations or financial involvement with any organization or entity with a financial interest in or financial conflict with the subject matter or materials discussed in the manuscript apart from those disclosed.

No writing assistance was utilized in the production of this manuscript. 


\section{Ethical conduct of research}

The authors state that they have obtained appropriate institutional review board approval or have followed the principles outlined in the Declaration of Helsinki for all human or animal experimental investigations. In addition, for investigations involving human subjects, informed consent has been obtained from the participants involved.

Data collection by chart review was approved by the institutional review board of the University Medical Center Groningen.

\section{Open access}

This work is licensed under the Attribution-NonCommercial-NoDerivatives 4.0 Unported License. To view a copy of this license, visit http://creativecommons.org/licenses/by-nc-nd/4.0/

\section{References}

Papers of special note have been highlighted as: • of interest; $\bullet \bullet$ of considerable interest

1. Balch CM, Gershenwald JE, Soong SJ et al. Multivariate analysis of prognostic factors among 2313 patients with stage III melanoma: comparison of nodal micrometastases versus macrometastases. J. Clin. Oncol. 28(14), 2452-2459 (2010).

2. Hodi FS, O’Day SJ, McDermott DF et al. Improved survival with ipilimumab in patients with metastatic melanoma. N. Engl. J. Med. 363(8), 711-723 (2010).

3. Chapman PB, Hauschild A, Robert C et al. Improved survival with vemurafenib in melanoma with BRAF V600E mutation. N. Engl. J. Med. 364(26), 2507-2516 (2011).

4. Long GV, Stroyakovskiy D, Gogas $\mathrm{H}$ et al. Combined BRAF and MEK inhibition versus BRAF inhibition alone in melanoma. N. Engl. J. Med. 371(20), 1877-1888 (2014).

5. Robert C, Long GV, Brady B et al. Nivolumab in previously untreated melanoma without BRAF mutation. N. Engl. J. Med. 372(4), 320-330 (2015).

6. Robert C, Schachter J, Long GV et al. Pembrolizumab versus ipilimumab in advanced melanoma. N. Engl. J. Med. 372(26), 2521-2532 (2015).

7. Davies H, Bignell GR, Cox C et al. Mutations of the BRAF gene in human cancer. Nature 417(6892), 949-954 (2002).

8. Hauschild A, Grob JJ, Demidov LV et al. Dabrafenib in BRAF-mutated metastatic melanoma: a multicentre, open-label, Phase III randomised controlled trial. Lancet 380(9839), 358-365 (2012).

9. Sosman JA, Kim KB, Schuchter L et al. Survival in BRAF V600-mutant advanced melanoma treated with vemurafenib. N. Engl. J. Med. 366(8), 707-714 (2012).

10. National Institutes of Health and National Cancer Institute: Common Terminology Criteria for Adverse Events 2016. (2010). https://ctep.cancer.gov/protocoldevelopment/electronic_applications/ctc.htm\#ctc_40

11. Fisher B, Bryant J, Wolmark $\mathrm{N}$ et al. Effect of preoperative chemotherapy on the outcome of women with operable breast cancer. J. Clin. Oncol. 16(8), 2672-2685 (1998).

12. Shapiro J, van Lanschot JJ, Hulshof MC et al. Neoadjuvant chemoradiotherapy plus surgery versus surgery alone for oesophageal or junctional cancer (CROSS): long-term results of a randomised controlled trial. Lancet Oncol. 16(9), 1090-1098 (2015).

13. Moschos SJ, Edington HD, Land SR et al. Neoadjuvant treatment of regional stage IIIB melanoma with high-dose interferon $\alpha-2 b$ induces objective tumor regression in association with modulation of tumor infiltrating host cellular immune responses. J. Clin. Oncol. 24(19), 3164-3171 (2006).

14. Kruijff S, Bastiaannet E, Brouwers AH et al. Use of S-100B to evaluate therapy effects during bevacizumab induction treatment in AJCC stage III melanoma. Ann. Surg. Oncol. 19(2), 620-626 (2012).

15. Koers K, Francken AB, Haanen JB, Woerdeman LA, van der Hage JA. Vemurafenib as neoadjuvant treatment for unresectable regional metastatic melanoma. J. Clin. Oncol. 31(16), e251-e253 (2013).

16. Rastrelli M, Pigozzo J, di Maggio A, Tosi AL, Sileni VC, Rossi CR. Neoadjuvant treatment with dabrafenib of unresectable localizations from occult melanoma. Melanoma Res. 24(4), 413-414 (2014).

17. Fadaki N, Cardona-Huerta S, Martineau L et al. Inoperable bulky melanoma responds to neoadjuvant therapy with vemurafenib. BMJ Case Rep. doi:10.1136/bcr-2012-007034 (2012) (Epub ahead of print).

18. Melnik I, Lotem M, Yoffe B. A new role of vemurafenib as a neoadjuvant treatment of axillary and brain melanoma metastases. Case Rep. Oncol. Med. doi:10.1155/2013/794239 (2013) (Epub ahead of print).

19. Sloot S, Zager JS, Kudchadkar RR et al. BRAF inhibition for advanced locoregional BRAF V600E mutant melanoma: a potential neoadjuvant strategy. Melanoma Res. 26(1), 83-87 (2016).

20. Johnson AS, Crandall H, Dahlman K, Kelley MC. Preliminary results from a prospective trial of preoperative combined BRAF and MEK-targeted therapy in advanced BRAF mutation-positive melanoma. J. Am. Coll. Surg. 220(4), 581.e1-593.e1 (2015).

21. Long GV, Weber JS, Infante JR et al. Overall survival and durable responses in patients with BRAF V600-mutant metastatic melanoma receiving dabrafenib combined with trametinib. J. Clin. Oncol. 34(8), 871-878 (2016). 
-• Points out that durable responses to BRAF inhibitors are possible and long-term survival can be achieved.

22. Ribas A, Gonzalez R, Pavlick A et al. Combination of vemurafenib and cobimetinib in patients with advanced BRAF(V600)-mutated melanoma: a Phase Ib study. Lancet Oncol. 15(9), 954-965 (2014).

23. Chapman $\mathrm{PB}$, Hauschild A, Robert $\mathrm{C}$ et al. Improved survival with vemurafenib in melanoma with BRAF V600E mutation. N. Engl. J. Med. 364(26), 2507-2516 (2011).

24. Robert C, Karaszewska B, Schachter J et al. Improved overall survival in melanoma with combined dabrafenib and trametinib. N. Engl. J. Med. 372(1), 30-39 (2015).

25. Ogston KN, Miller ID, Payne $\mathrm{S}$ et al. A new histological grading system to assess response of breast cancers to primary chemotherapy: prognostic significance and survival. Breast 12(5), 320-327 (2003).

26. Henderson MA, Burmeister BH, Ainslie J et al. Adjuvant lymph-node field radiotherapy versus observation only in patients with melanoma at high risk of further lymph-node field relapse after lymphadenectomy (ANZMTG 01.02/TROG 02.01): 6-year follow-up of a Phase III, randomised controlled trial. Lancet Oncol. 16(9), 1049-1060 (2015).

27. Eggermont AM, Chiarion-Sileni V, Grob JJ et al. Adjuvant ipilimumab versus placebo after complete resection of high-risk stage III melanoma (EORTC 18071): a randomised, double-blind, Phase III trial. Lancet Oncol. 16(5), 522-530 (2015).

28. Eggermont AM, Chiarion-Sileni V, Grob JJ et al. Prolonged survival in stage III melanoma with ipilimumab adjuvant therapy. N. Engl. J. Med. 375(19), 1845-1855 (2016).

- Points out that survival improves when patients are treated adjuvantly with immunotherapy. 